\title{
AUTOMATING THE CREATION OF FACILITY AND ENERGY MANAGEMENT BUILDING INFORMATION MODELS
}

\author{
Brandon Bortoluzzi ${ }^{1}$, Daniel Sobieraj ${ }^{2}$, and J.J. McArthur ${ }^{3}$
}

\begin{abstract}
Building Information Models (BIMs) are widely recognized as being valuable asset management tools, however the resources required to develop BIMs of existing buildings for Facilities Management (FM) purposes are a recognized barrier to entry. Significant developments have been made for generating geometrically complex models using scanning technologies, however the resultant models are often extremely large, requiring significant computational resources. This paper presents an automated process that uses 2D floorplans and elevation drawings to generate semantically-rich, BIMs with adequate geometry for energy simulation and integration of semantic data of specific value in day-to-day building operations management. The proposed approach is limited to the information available regarding the building and develops a model requiring minimal resources to both develop and maintain, while providing the flexibility for incorporating complex geometry when such information becomes available.

A case study of a university campus is presented where 20 buildings were modelled using available 2D architectural CAD files (floorplans and elevations) to demonstrate and evaluate this approach. Process speed, accuracy, and resultant model quality are discussed, along with automation process limitations.
\end{abstract}

Keywords: building information modelling, automation, case study, existing buildings, facility management

\section{INTRODUCTION}

Building Information Modelling is transforming how buildings are designed, constructed, and managed through their life cycle. Of these three areas, its application to facility management is limited, particularly in the case of existing buildings (Volk, et al., 2014) (Eadie, et al., 2013) where the major barrier to adoption is the creation of Building Information Management models (BIMs) for Facility Management (BIM-FMs), despite research demonstrating both time and financial benefits to the use of BIM in this context (Volk, et al., 2014) (Love, et al., 2014) (Bryde, et al., 2013).

Previous work (McArthur, 2015) has challenged the assumption that as-built BIM-FMs are required for day-to-day facility operations; instead a simplified geometry can host the semantic information to allow the BIM-FM to serve as a common data environment. To support this approach and enable portfolio owners to quickly develop BIM-FMs, this paper

M.Arch Candidate, Department of Architectural Science, Ryerson University, Toronto, Canada brandon.bortoluzzi@ryerson.ca

2 B.ArchSci Candidate, Department of Architectural Science, Ryerson University, Toronto, Canada daniel.sobieraj@ryerson.ca

3 Assistant Professor, Department of Architectural Science, Ryerson University, Toronto, Canada, jimcarthur@ryerson.ca 
presents an automated process to generate BIM-FMs from vector floorplans and reduce this barrier to adoption.

The paper first presents a review of BIM in FM and existing automation processes through the lens of the day-to-day operations, followed by an overview of the BIM-FM model creation process. This process was then used to generate BIM-FMs for 20 additional buildings across Ryerson University in a case study, which is presented and evaluate. Finally, the results of this cases study are discussed, and limitations of this approach and potential research directions are identified.

\section{BIM FOR EXISTING BUILDINGS}

In the FM context BIM has been under adopted in comparison to the adoption in the design and construction phases (Bryde, et al., 2013) (Eadie, et al., 2013). Despite this there are known benefits such as; utility cost reductions, comfort management, space optimization, improved inventory management, etc. when BIM-FM is implemented (Love, et al., 2014). The barriers to this adoption are fairly widespread but include high level of effort, data transfer between multiple systems, and the incompleteness of data (Volk, et al., 2014). Significant research activity is aimed to address systems issues and test new strategies for developing FM-ready BIM, e.g. (Kiviniemi \& Codinhoto, 2014; Brooks \& Lucas, 2014). Technologies such as cloud computing (Jiao, et al., 2013), passive RFID tags (Lin, et al., 2014) and 3D scanning (Laing, et al., 2015) have facilitated adoption and added value to BIM in a Facility Management/Operations context, and the richness of information in BIM-FMs and the ability to access this information on mobile devices offer advantages over traditional FM software (Watson, 2011), however the application of such techniques in existing buildings is limited.

\subsection{Building Information Model Generation for Existing Buildings}

Previous research has shown that BIMs need to be tailored to end-uses; not one-size fits all (Giminez, 2015)

Recent studies have investigated means of generating BIMs for existing buildings, as summarized by $\mathrm{Lu}$ and Lee (2015) and Pătrăucean et al. (2015). A complete as-built building information model is considered by many a requirement BIM use in operations and the associated level of effort poses a significant barrier to the use of BIM in this context (Volk, et al., 2014). While 3D laser scanning and scan-to-BIM technologies have developed significantly (Laing, et al., 2015), their outputs are generally limited to visible elements, results in extremely large point cloud models, and thus does not fully resolve this issue.

Defining appropriate parameters in BIM, mapping CAFM data (from spreadsheet format) to BIM using parametric tools such as Dynamo where plug-ins aren't available, and developing the BIM itself all require significant effort, and this level of investment is a contributor to the slow adoption of BIM in the FM context (Volk, et al., 2014). Very few papers have investigated automating data transfer from CAFM systems, though two case studies have been presented in (Lazar \& McArthur, 2016; Khaja, et al., 2016).

Automated generation of BIMs from 2D drawings is a subject of increasing research. Giminez et al. (2016) review other automated generation processes to date and present a process to convert simple 2D floorplans to 3D building models using $\mathrm{C}++$ and a mathematical approach to identify wall elements and assign them to the associated room. This was demonstrated effective for simple geometries but could not integrate more complex elements such as curved facades, varying building height, or roof elements. 


\section{METHOdology}

The methodology included in this paper was developed with an aim to model the vast majority of existing buildings and while it was developed specifically using Autodesk products (AutoCAD, Revit, Dynamo), the process can be similarly applied to other platforms. Where specific product functionality is called out, it is italicized. Limitations on the complexity of buildings that this process can accommodate are discussed at the end of the paper as well as within the appropriate section for each step. The overall BIM-FM model creation process is shown schematically in Figure 1 below. Note that this process has been developed specifically to develop models to support day-to-day building operations rather than renovation and is not intended to provide comprehensive geometric information. Where as-built conditions are reflected in discipline CAD drawings (e.g. structural, mechanical, and electrical), these may be incorporated as references at the end of the process to avoid introducing error.

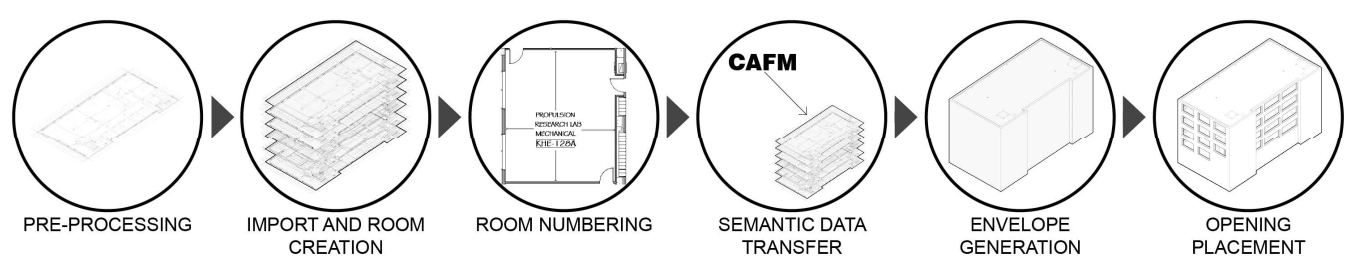

Figure 1 BIM-FM Creation Process Overview

\subsection{Pre-Processing}

This process and the pre-processing phase assumes that 2D floorplans - preferably in a vector file format - at minimum are available for each floor of the building. Where the existing drawing files for the buildings are not in a CAD format they must be translated and saved as .dwg files as part of pre-processing. Files are then checked to ensure that room boundaries and envelope component linework consist of closed curves, segregated onto the specified layers illustrated in Figure 2a. A similar approach to define elevation linework by layer is shown in Figure $2 \mathrm{~b}$. Where elevations are not available, a semantically-rich model can be created without modelling the building envelope.

a)

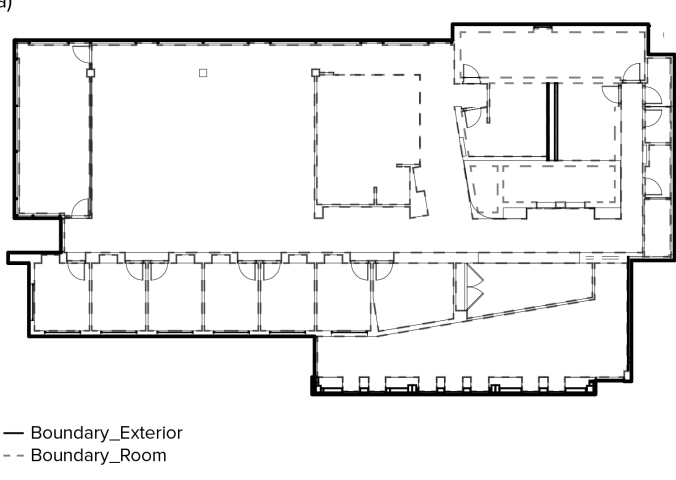

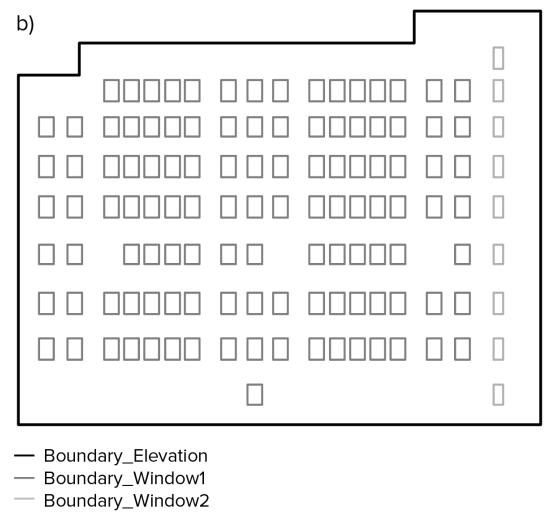

Figure 2 Layer definitions in pre-processing of a) floorplans (left) and b) elevations (right)

\subsection{Room Creation}

Room elements are used to host the static, semantic building information, and the dynamic FM data attributable to spaces and thus form the core of the BIM-FM model. These rooms 
are placed using the floorplans as a guide by first defining building levels (slab-to-slab heights informed by available elevation drawings) and the associated floorplan filenames in a spreadsheet file. An automated script (Dynamo graph) uses this information to import these files to the correct levels. Where as-built drawings are available, these may be also incorporated later in this process using a similar approach, but this is not recommended at this stage as it introduces unnecessary complexity to the model that may introduce error. A quality assurance process occurs at this stage to avoid propagation of errors in the subsequent automation.

Next, room elements are created level by level. The imported floorplan (CAD file) is selected, room boundary layer ("boundary_room") identified, and the desired BIM linetype specified. The algorithm (Revit $A P I$ ) then loops through each curve segment of the specified layer and adds it to a curve array, which is then used to define a set of room separation lines. In the case study presented, this was executed using a published macro (Kilkenny, 2015), however this process can be adapted to any platform. Within Revit, the Place Rooms Automatically command automates the next step in this process, which is to define each area bound by separation lines as a schedulable room.

\subsection{Room Numbering}

To automatically number the rooms, another script (Dynamo graph) creates a list of room tags and the insertion point for each is found and added to an array. One insertion point at a time, the XY coordinates are compared with the room boundaries and a k-nearest neighbour algorithm $(\mathrm{k}=1)$ is used to identify the room containing the insertion point. The text in the room tag linework is then extracted from the room tag element and assigned as the room name parameter in the BIM. A limitation of this algorithm is that room tags placed outside the room (e.g. for a small closet), cannot be automated and can result in the mislabelling of larger room hosting multiple room tags. As a result, quality assurance must also occur at this stage before proceeding to CAFM data integration and envelope modelling.

\subsection{Room Semantic Information}

Due to the wide range of systems used within the realm of facilities management, the BIM becomes an important common data environment for information from multiple sources. Where BIM-compatible CAFM systems are in place, no further automation is required to transfer FM information, but where this is not the case, CAFM data can be imported into custom parameters defined in the BIM using room codes as the unique object identifiers. In this case study, this was achieved using a series of scripts (Dynamo graphs) used to update the BIM with space management data (room ownership, office assignment, etc.) and work request and occupant complaint histories. This latter body of work has been described in a previously published paper (Khaja, et al., 2016).

\subsection{Envelope Generation}

At the beginning of this step, the BIM is highly semantic, with minimal geometry, namely level definitions and referenced floorplan line elements, but has no true 3D geometric element. To expand the functionality of such a model to permit energy and solar analysis, the envelope must be created and defined.

Generation of the envelope requires the application of two automated processes: the definition of exterior boundaries; and the creation of wall elements. The envelope boundary is defined using a similar process to the room boundary however substitutes the room separation line type with the model line type. The wall elements are generated using 
a script (Dynamo graph) which locates the level of the boundary element and generates a user specified wall type (must be developed and included in the BIM wall types in advance) between the identified level and the one above it.

It is important to note that while a simple level to level extrusion (Wall.ByCurveandLevels in Dynamo) works for most simple mass buildings of the urban context, a slightly different graph utilizing lofts (Wall.ByFace) has also been developed, though not yet tested to the same standard, for more complex forms.

\subsection{Automated Placement of Openings}

The placement of openings in the façade is the final required step to allow for the analysis functionality outlined in the previous section. This process requires the use of three steps: the import of previously imported CAD elevations, the automated translation of the linework to BIM line types and the running of a script to place the appropriate openings at each identified location.

The import of the drawings must be done in a way that allows them to operate on their own reference planes in the appropriate elevation views of the building. They can either be imported into a common view such as a plan or 3D view before being rotated and assigned to a reference plane or imported directly to the corresponding elevation view and then assigned to the reference plane. In either case a QA process must be carried out to ensure the alignment of the elevations with the building envelope developed in the previous section. The linework from the imported file is next converted to native linetypes using a script similar to that used for room creation. The resultant elements are extruded through the destination elevation, the intersection of which defines the wall opening, and the centroid of this opening will be used for placement of the appropriate window or door family. Multiple linetypes are used to differentiate the elements in the most suitable way for a particular building. Where the openings are consistent (e.g. a single window family for the majority of each façade) each elevation is differentiated with a different linetype and the extrusion vectors are defined based on the source lineweights, allowing all facades to be processed in a single step. In more complex buildings with multiple opening types on each façade, a second script was developed that uses these lineweights to distinguish each opening type, requiring the processing of each elevation separately.

\section{Evaluation}

An ongoing BIM in Sustainable Operation pilot project has been underway since October 2014 to develop a virtual campus model for Ryerson University. This model consists of BIM-FMs for each individual building nested within a site model created using GIS data. Up until the automated process was deployed in June 2016, only nine buildings (1.35 million sf) had been modeled. The automated process presented previously has been used to generate 20 additional buildings with a total 115 levels making up the centre of the campus.

On average, buildings took an average of 8 hours to pre-process floorplans and elevations, ranging from 1-16hrs; buildings with more complex layouts and larger numbers of storeys taking the longer duration. Once pre-processed, the floorplan import, room identification, labelling and massing took an average of 35 minutes per buildings, varying from 6 to 90 minutes, scaling roughly with the number of floorplans in the building. Opening placement required an average of 2 hours per building and did not scale linearly with building height but rather with the diversity of opening types and number of wall faces, since each had to be individually addressed. This latter issue increased the 
processing time substantially for buildings with complex façades as the script had to be rerun multiple times to capture the openings for each facade.

The automated algorithm was unable to place openings correctly on some curved and non-vertical façade elements, requiring manual manipulation of the model was necessary to match the as-constructed condition. The Engineering building (Figure 3a) required manual adjustment of the extruded wall elements to generate the actual angled façade condition as well as manual opening placement. The position of the openings on these surfaces were also inaccurate due to the non-orthogonal projection. A second case involved a university residence building (Figure $3 \mathrm{~b}$ ) with a large curved façade. This façade was accurately generated, but the openings could not be placed as the software was unable to project onto this curved surface, and thus manual placement was required. In each case, the manual step increased the overall processing time by approximately two hours. Buildings where the above conditions did not exist were developed entirely using the process presented and a selection of these are illustrated in Figures 3c-f.

a)

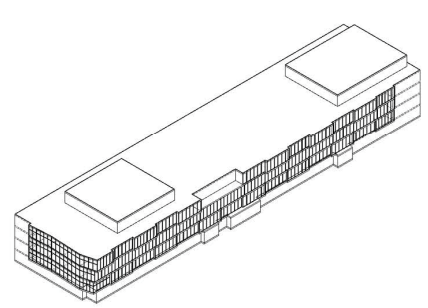

d)

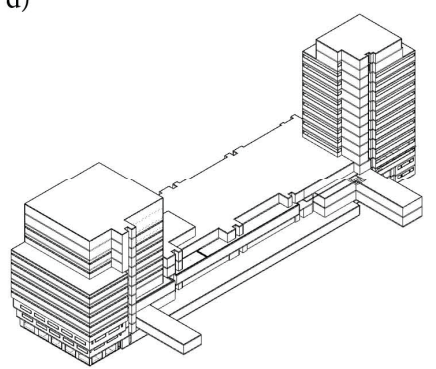

b)

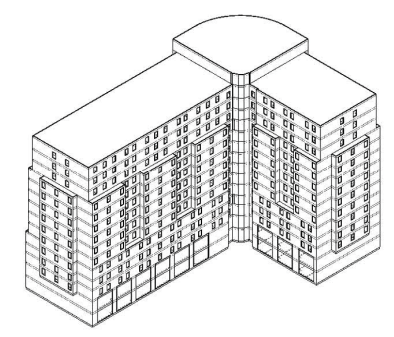

e)

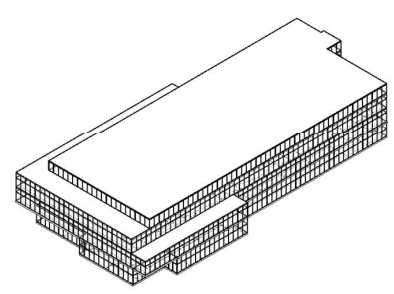

c)

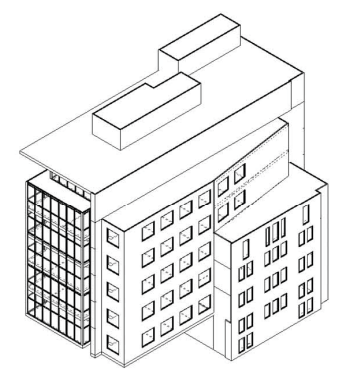

f)

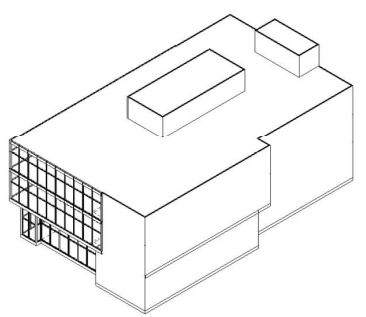

Figure 3 Selected buildings created using the automated algorithm

The generated models were generally of high quality, with all elements bound and mapped to the correct material properties, however errors arose through three assumptions. First, split levels are not accounted for in this algorithm and each floorplate is assumed to occur at a single elevation. While this was correct for the majority of cases, this was an oversimplification for some buildings, evident particularly when elevations were applied to the wall faces and openings did not align. Second, when the elevation files were unavailable at the start of the process, levels heights were assumed. Once CAD elevations were generated, errors in such assumptions were obvious and required adjustment of the level heights prior to opening extrusion to avoid error. Finally, the envelope for the sub-grade levels could not be generated using the algorithm as-presented. Further, the presence of underground levels introduced error, requiring that any belowgrade levels and associated rooms be generated at after this process. 


\section{DISCUSSION AND CONCLUSIONS}

This case study demonstrates the potential of the proposed automation process to streamline the development of simplified BIMs for FM applications. Industry applications are significant as this provides a basis for BIM-FM generation with significantly reduced input; at Ryerson, the automated models were well-received by the Facilities team, who found them adequate for CAFM data mapping and visualization.

The key benefit of this process is the decrease in modelling time. The first batch of buildings were taking 100-250 hours to complete to the level presented with an average 25 hours per storey. In this study, the automation script reduced this to 1.3 to 2.6 hours per storey, resulting in an average time savings of $90-95 \%$. As noted previously, the majority $(75 \%)$ of the time required was used to manually clean up the 2D files. There is significant opportunity to improve this process by automating the drawing pre-processing. This is a topic of future research using AutoLISP and - based on exploratory tests - the authors believe that this may reduce the pre-processing time by up to $90 \%$.

The creation and tagging of rooms and data integration steps each take minimal time to execute $(<5 \mathrm{~s})$. The integration of all of these steps into a single script was considered, however they have been kept separate in order to force the user to check the model for errors and prevent their propagation between these steps. The most common quality issues observed include: misalignment of imported 2D drawings based on inconsistent origin points in the original files, incorrect drawing scaling due to poor unit recognition by the BIM software, and the confounding of room names and numbers when multiple room tags co-exist within a larger room (e.g. smaller rooms tagged with leaders). Pattern recognition and other analytic techniques may offer the ability to automate this checking process and further streamline this set of steps, however the time saved is small compared to potential improvements in pre-processing and is not currently being pursued.

The creation of walls and placement of openings, presented the most significant challenges and provide the greatest opportunity for refinement of the automation process. During wall, the presence of basements was a significant limitation with the current script because the software used for the case study (Revit) was unable to order levels from the lowest to the highest; instead ordering was based on the level creation order, starting by default with the ground floor. To overcome this, basement levels were excluded from the files for the model creation steps and imported only after the wall element geometry was generated. A separate envelope generation script for subgrade floors will be required to model such elements. A second limitation is that the extrusion approach used for wall generation is inappropriate for some geometry and additional scripts for lofting and other 3D geometry creation are under development to expand the applicability of this process. For complex geometry, significant research is required to recognize each pattern from plan and elevation views. In the interim, manual selection of each script (extrude, loft, etc.) is required. Third, the automation process places a single wall type and will be expanded to allow multiple types, improve flexibility. Finally, opening placement on non-rectilinear surfaces suffered from the incorrect placement due to inaccurate identification and projection of the opening centroids and will be addressed in future research.

\section{ACKNOWLEDGMENTS}

This project is funded by the Big Data Research, Analytics, and Information Network (BRAIN) Alliance established by the Ontario Research Fund - Research Excellence 
Program (ORF-RE), along with Ryerson University (Work-Study Research Assistant grant, Career Boost, and Dean's Research Fund).

\section{REFERENCES}

Brooks, T. \& Lucas, J., 2014. A Study to Support BIM Turnover to Facility Managers for Use after Construction. Computing in Civil and Building Engineering, 243-250.

Bryde, D., Broquetas, M. \& Volm, J. M., 2013. The project benefits of building information modelling (BIM). International Journal of Project Managmeent, 31(7), 971-980.

Eadie, R. et al., 2013. BIM implementation throughout the UK construction project lifecycle: an analysis. Automation in Construction, 36, 145-151.

Giminez, L., Robert, S., Suard, F. \& Zreik, K., 2016. Automatic reconstruction of 3D building models from scanned 2D floor plans.. Automation in Construction, 63, 48-56.

Jiao, Y. et al., 2013. A cloud approach to unified lifecycle data management in architecture, engineering, construction and facilities management: Integrating BIMs and SNS. Advanced Engineering Informatics, 27(2), 173-188.

Khaja, M., Seo, J. D. \& McArthur, J. J., 2016. Optimizing BIM Metadata Manipulation Using Parametric Tools. Tempe, s.n., pp. 259-266.

Kilkenny, M., 2015. DWG to Revit Lines (Macro). New York: ArchSmarter.

Kiviniemi, A. \& Codinhoto, R., 2014. Challenges in the Implementation of BIM for FM Case Manchester Town Hall Complex. Computing in Civil and Building Engineering, pp. 665-672.

Laing, R., Leon, M., Isaacs, J. \& Georgiev, D., 2015. Scan to BIM: the development of a clear workflow for the incorporation of point clouds within a BIM environment.. Bristol, UK, WIT Press, pp. 297-307.

Lazar, S. \& McArthur, J. J., 2016. BIM for Corporate Real Estate Data Visualization from Disparate Systems.. Lecture Notes of the Institute for Computer Sciences, Social Informatics and Telecommunications Engineering, 166, 504-516.

Lin, Y., Su, Y. \& Chen, Y., 2014. Developing Mobile BIM/2D Barcode-Based Automated Facility Management System. The Scientific World Journal, p.374735.

Love, P. et al., 2014. A benefits realization management building information modeling framework for asset owners. Automation in Construction, 37, 1-10.

Lu, Q. \& Lee, S. H., 2015. Comparative analysis of technologies and methods for automatic construction of building information models for existing buildings.. Hong Kong, Institution of Civil Engineers.

McArthur, J., 2015. A building information management (BIM) framework and supporting case study for existing building operations, maintenance and sustainability. Chicago, USA, Elsevier, pp. 1104-111.

Pătrăucean, V. et al., 2015. State of research in automatic as-built modelling.. Advanced Engineering Informatics, 29(2), 162-171.

Volk, R., Stengel, J. \& Scultmann, F., 2014. Building Information Modeling (BIM) for existing buildings - Literature review and future needs. Automation in Construction, 38, 109-127.

Watson, A., 2011. Digital buildings - challenges and opportunities. Advanced Engineering Informatics, 25, 573-581. 